\title{
CAMBIOS EN LA MORTALIDAD POR EVENTOS CLIMÁTICOS EXTREMOS EN MÉXICO ENTRE EL 2000 Y 2015
}

\author{
José Alfredo Jáuregui Díaz ${ }^{1}$, María de Jesús Ávila Sánchez ${ }^{1, *}$ y Rodrigo Tovar Cabañas ${ }^{1}$
}

\section{RESUMEN}

El presente trabajo tiene el objetivo de examinar los cambios en la mortalidad inducida por varios eventos climáticos extremos de 2000 a 2015 en México y analizar las características de las víctimas, así como las vulnerabilidades demográficas y geográficas para el desarrollo de estrategias adaptativas y preventivas para geografías y grupos poblacionales específicos para minimizar los efectos del clima extremo. El estudio se basa en el análisis estadístico de microdatos proporcionados por diferentes registros administrativos. Los resultados muestran que la mortalidad por eventos extremos de este tipo continúa siendo inaceptablemente alta, ya que la mayoría de esas muertes podrían haberse evitado. La letalidad de los desastres se produce no solo por la exposición a una determinada amenaza, sino también, por la vulnerabilidad acumulada en que se encuentran determinadas poblaciones. Teniendo en cuenta los resultados de la investigación, los programas de prevención deben dirigirse a los hombres en edades productivas y adultos mayores, a las mujeres en particular a las niñas menores de nueve años y adultas mayores, lo que reduciría el impacto sobre la mortalidad por desastres relacionados al clima extremo.

\section{PALABRAS CLAVE}

Desastres, Vulnerabilidad, Mortalidad, Clima, México

\section{CHANGES IN MORTALITY BY EXTREME WEATHER EVENTS IN MEXICO BETWEEN 2000 AND 2015}

\section{ABSTRACT}

This document aims to examine the changes in morality induced by several extreme weather events from 2000 to 2015 in Mexico and analyze the characteristics of the victims. This analysis approaches the demographic and geographical vulnerabilities of the affected population for the development of adaptive and preventive strategies that minimize the effects of extreme weather. The study is based on the statistical analysis of microdata provided by different administrative records. The results show that mortality from these extreme events remains unacceptably high, since most of these deaths could have been prevented. The lethality of disasters occurs not only due to exposure to a certain threat, but also due to the accumulated vulnerability of certain populations. Taking this into account, the results of this research suggest that targeted prevention programs should focus on male in labor ages and male elderly, and female with particular emphasis on girls under nine years old and female elderly. This may help to reduce the impact on mortality due to disasters triggered by extreme weather events.

\section{KEYWORDS}

Disasters, Vulnerability, Mortality, Weather, Mexico
1. Instituto de

Investigaciones Sociales, Universidad Autónoma de Nuevo León, Monterrey, México.

*Autor de correspondencia: marycolef@yahoo.com

\section{RECIBIDO}

24 de junio de 2019

\section{ACEPTADO}

1o de octubre de 2019

\section{PUBLICADO}

1 de enero de 2020

\section{Formato cita}

Recomendada (APA): Jáuregui Díaz, J.A., Ávila Sánchez M.J. \& Tovar Cabañas, R. (2020). Cambios en la Mortalidad por Eventos Climáticos Extremos en México entre el 2000 y 2015. Revista de Estudios Latinoamericanos sobre Reducción del Riesgo de Desastres REDER, 4(1), pp.8o-94.

\section{(ㄷ) $(1) \Theta$}

Todos los artículos publicados en REDER siguen una política de Acceso Abierto y se respaldan en una Licencia CreativeCommons Atribución-NoComercial 4.0 Internacional.

Revista de Estudios

Latinoamericanos sobre Reducción del Riesgo de Desastres (REDER)

Diseño: Lupe Bezzina Tipografía: Hospital 


\section{INTRODUCCIÓN}

México es una región altamente expuesta a múltiples amenazas de origen natural-sísmica e hidro-meteorológica y socio-natural-, dichas amenazas se están incrementando debido al cambio climático, que intensifica los fenómenos relacionados con las lluvias intensas y sequías prolongadas, eventos con un impacto inmediato y a largo plazo en las poblaciones más vulnerables.

Las proyecciones realizadas hasta la fecha sugieren que México se verá severamente afectado por el cambio climático global, con variaciones importantes en la temperatura y precipitación promedio anual durante este siglo (McCarthy, 2001; Emanuel 2005; INE-Semarnat, 2006; Magrin et al. 2007), en combinación con tormentas tropicales y sequías de mayor intensidad (INE-Semarnat, 2006; Rojas-Rodríguez \& Hurtado-Díaz, 2006; Magrin et al. 2007).

La mortalidad es la expresión máxima de desastre. Según señala la Red de Estudios Sociales en Prevención de Desastres en América Latina (2018) durante el último medio siglo, los desastres han producido en México más de 17 millones de damnificados, otros siete millones de heridos, más de 50 mil muertos y 10 mil desaparecidos.

Estas cifras nos recuerdan el fuerte impacto que los desastres ejercen sobre la vida y el desarrollo de las distintas regiones del país, sin embargo, la investigación sobre el impacto de las muertes relacionadas con eventos climáticos extremos es bastante limitada. Los estudios de daños causados por desastres meteorológicos generalmente abordan la pérdida económica y pocas veces examinan los factores sociales de los desastres en relación con las víctimas y los grupos vulnerables. Una excepción es lo hecho por Noji (2000), quien analizó las tasas de mortalidad por tipo de desastre, pero a nivel mundial, otros casos aislados son: Arcos et al. (2002), quienes estudiaron las medidas de frecuencia, tanto en la morbilidad y mortalidad por tipo de desastre, pero a nivel mundial.

El presente documento tiene como objetivo examinar los cambios en la moralidad inducida por varios eventos climáticos extremos de 2000 a 2015 en México y analizar las características de las víctimas por sexo, edad, escolaridad, ocupación y las diferencias regionales. Se utilizarán los datos sobre el número de muertes por desastres meteorológicos recabados por la Secretaría de Salud y del Instituto Nacional de Geografía e Informática (SSA-INEGI).

Los resultados de este estudio contribuirán al desarrollo de estrategias adaptativas y preventivas para geografías y grupos poblacionales específicos para minimizar los efectos del clima extremo.

\section{MARCO TEÓRICO: LOS DESASTRES METEOROLÓGICOS Y LAS CIENCIAS SOCIALES}

Desde el punto de vista del aporte que las ciencias sociales hacen a los estudios de los desastres en América Latina, existe una ausencia de estudios sobre la naturaleza social de los desastres, debido al dominio que ejercen las ciencias naturales y básicas sobre la problemática de los desastres en Latinoamérica es casi total.

La sociedad no aparece en la fórmula, ni como objeto de estudio, ni como objeto de acción y cambio en cuanto sus patrones de comportamiento y de incidencia en la concreción de situaciones de desastre. Las razones para esa falta de atención incluyen, entre otras:

- Lo raro, impredecible y súbito de la ocurrencia de un desastre;

- La concepción de que el comportamiento de la naturaleza y su impacto no se pueden controlar:

- La dificultad para conseguir datos útiles sobre las consecuencias de los desastres durante su desarrollo e inmediatamente después.

Los desastres no se conciben como una parte integral del espectro de relaciones hombrenaturaleza o dependientes directamente de ellos, sino más bien como un 'problema superado', temporal y territorialmente limitados, algo raro o extraordinario, 'eventos que violan la vida normal y sus relaciones con el hábitat'.

El interés en los desastres por los estudiosos de las ciencias sociales ha sido pasajero y casi obligado por la importancia o visibilidad del evento de gran magnitud (por ejemplo, el terremoto de México en 1985), por lo que no ha sido reconocido como un tema genérico de estudio permanente en las ciencias sociales (una excepción son las líneas de trabajo de Medrano y García (2014), quienes 
desde el siglo pasado ininterrumpidamente han trabajado el estudio de los desastres agrícolas y climatológicos). Un paso necesario para la construcción de la relación entre las ciencias sociales y desastre es la conceptualización social del desastre, es decir las características que lo definen y las condiciones necesarias para que exista.

Los desastres tienen un impacto en el territorio caracterizado por una estructura social vulnerable y donde la diferenciación interna de la sociedad influye en forma importante en los daños sufridos y en los grupos sociales afectados en mayor o menor grado. Desde esta perspectiva, un desastre es tanto producto como resultado de procesos sociales, histórica y territorialmente circunscritos y conformados.

Puede definirse un desastre como: Una ocasión de crisis o estrés social, observable en el tiempo y el espacio, en que sociedades o sus componentes (comunidades, regiones, etc.) sufren daños o pérdidas físicas y alteraciones en su funcionamiento rutinario, alterando el funcionamiento de la comunidad humana afectada y excediendo su capacidad para hacer frente a la situación utilizando sus propios recursos. Tanto las causas como las consecuencias de los desastres son producto de procesos sociales que existen en el interior de la sociedad (Lavell, 1993; EIRD, 2007).

\section{La vulnerabilidad un concepto ligados al estudio del desastre}

La vulnerabilidad de la población y su exposición al riesgo de sufrir algún desastre difieren sustancialmente según los subgrupos de población debido a algunas características sociodemográficas como la edad, el género, la etnia, nivel educativo y otras de tipo contextual como la religión. Además, abrían que sumar las susceptibilidades fisiológicas del espacio geográfico que se habita, factores socioeconómicos, psicosociales, exposición y percepciones al riesgo, y la capacidad de respuesta.

La vulnerabilidad diferencial está determinada por las características demográficas socialmente construidas y las normas asociadas a estas características. Por ejemplo, las mujeres exhibieron una mayor susceptibilidad a las olas de calor (Michelozzi, et al. 2005). En cambio, los hombres tienen mayores riesgos de mortalidad por inundaciones y tormentas que las mujeres, ya que los hombres participan más frecuentemente en actividades al aire libre y, por lo tanto, tienen mayor exposición a desastres hidrológicos (Zagheni et al., 2015).

El enfoque de vulnerabilidad ha sido destacado como clave en de desarrollo sostenible, tanto en la Cumbre Mundial sobre Desarrollo Sostenible de las Naciones Unidas en 2002 (Lutz \& Shah, 2002) como una década más tarde para RIO + 20 Cumbre de la Tierra (Lutz et al., 2012). En particular resulta necesario incorporar el concepto de vulnerabilidad diferencial demográfica en el análisis de la vulnerabilidad y las medidas políticas encaminadas a reducir la vulnerabilidad en consonancia con el Objetivo de Desarrollo Sostenible 10 sobre la igualdad para todos.

La vulnerabilidad de las localidades no se puede medir directamente en tiempo real, ya que los desastres suelen ser fenómenos complejos que se comportan, en la mayoría de los casos de forma estocástica debido a que las escalas espacio temporales de los sistemas involucrados (el físico y el social) suelen ser muy variadas y asimétricas (Kienberger, Blaschke \& Zaidi, 2013).

Se han relizado en diferentes latitudes geograficas enfuerzos por clasificar o tipificar las distintas vulnerabilidades con el propósito de ayudar a prevenir los desastres, a principios del siglo pasado Schmidtlein, Deutsch, Piegorsch y Cutter (2008), dividieron los tipos de vulnerabilidad en dos amplias categorías:

- Biofísica, referente a la exposición que cada persona tiene en particular a algún proceso externo;

- Social, ligada al entorno construido, a las políticas públicas y a la ecología humana. La vulnerabilidad social suele manifestarse cuando la carga ambiental (fenómenos geodinámicos tales como: tsunamis, inundaciones, flujos de lodo, tifones; entre otros) supera a cualquier tipo de sensibilidad genética y orden social.

En América Latina, Chaux (1998) intento entender la complejidad del enfoque, problematizó aún más el estudio de los desastres concluyendo que las vulnerabilidades de un sistema eran las amenazas de otro y viceversa, por ejemplo, los embalses hidroeléctricos suelen reducir la vulnerabilidad socioeconómica de las localidades distantes que se benefician de la llegada de suministro de energía eléctrica, pero, al mismo tiempo las localidades cercanas a dichos embalses 
hidroeléctricos incrementan su vulnerabilidad médica debido a los vectores de enfermedades zoonóticas tienen mayores espacios para su reproducción.

Otros autores han intentado ir más allá desarrollando algunos índices de vulnerabilidad social para comparar y evaluar la condición de vulnerabilidad social que manifiestan una o varias localidades en un contexto espacio-temporal (Cutter, Boruff \& Shirley, 2003). Sin embargo, se enfrentan a dificultades metodológicas, como: la necesidad de justificar los indicadores cuantitativos seleccionados para la confección del índice!

Es necesario tener en cuenta que cualquier métrica en torno a la vulnerabilidad depende de la disponibilidad de los datos, lo cual conlleva a una continua y sistemática elaboración de mediciones para cada lugar en particular (Chau et al., 2014). Por tal motivo es útil dar énfasis al análisis geoestadístico de algunas cualidades espaciales y de exposición a fuerzas de la naturaleza, como: tasa de mortalidad, tasa de damnificados, densidad de heridos por unidad de superficie; entre otros valores de carácter paramétrico, para geografizar e intronizar las investigaciones.

El análisis estadístico de determinado territorio ayuda a matizar el fenómeno de la vulnerabilidad social (Álvarez \& Cadena, 2006), ello se debe a que un índice conjunta una serie de variables, lo que permite valorar un conjunto de factores en una sola medición, la cual puede emplearse o ayudar a la toma de decisiones.

Dentro de los desastres la muerte es la expresión máxima del evento, en esta investigación se trabaja con todas las defunciones ocurridas en México durante el periodo 2000-2015 por alguna de las diez causas por exposición a fuerzas de la naturaleza, clasificadas de esa manera por la Organización Mundial de la Salud, y se enmarca en las ciencias emergentes abocadas al estudio de las relaciones entre la sociedad y la naturaleza.

Para reducir el riesgo de la población a sufrir la letalidad de los desastres es necesario identificar variables recurrentes, además es necesario incrementar la seguridad y la resiliencia de las comunidades. La letalidad de los desastres se produce no solo por la exposición a una determinada amenaza, sino también, por la vulnerabilidad acumulada en que se encuentran determinadas poblaciones.

\section{REFERENTES EMPÍRICOS SOBRE LAS MUERTES POR DESASTRES METEOROLÓGICOS}

Los desastres meteorológicos son un componente importante del cambio climático que afectan las tasas de mortalidad (Kovats, Campbell-Lendrum \& Matthies, 2005). Sin embargo, hay pocos estudios en ciencias sociales, como el de Kahn (2005), que evalúa las causas y características de las personas muertas por tipo de desastres a nivel mundial, aunque generalmente se abordan sólo las pérdidas económicas.

La mayoría de las investigaciones sobre eventos climáticos extremos se han centrado en una geografía específica, un desastre relacionado al clima o un periodo de tiempo particular. Por ejemplo, el estudio de Goklany (2009) examinó las tendencias en el número y la tasa de muertes para todos los tipos de desastres, como sequías, inundaciones, tormentas y huracanes para el periodo 1900 a 2008, concluyendo que las muertes por desastres muestran una tendencia decreciente.

A pesar de la tendencia a la baja en las víctimas humanas, los países son susceptibles a pérdidas significativas de vidas debido a los eventos climáticos extremos, como lo demuestra el huracán Katrina, la ola de calor en Europa en 2003, la ola de calor en Chicago en 1995 y el terremoto de la Ciudad de México en 2017.

En Estados Unidos destacan dos estudios, el primero elabora los patrones espaciales de la mortalidad por amenazas naturales a nivel de condado para los Estados Unidos de 1970 a 2004 utilizando una combinación de métodos geográficos y epidemiológicos (Borden \& Cutter, 2008); en tanto en el segundo se analizan diversos desastres durante más de 25 años, evaluando las características de sexo, raza y edad, así como las áreas vulnerables (Thacker, Lee, Sabogal \& Henderson, 2008).

Sin embargo, estos estudios no evaluaron las causas específicas de muerte según diversos tipos de desastres, por ejemplo, calor, rayo, inundaciones o tormentas como lo hicieron otros, que en los párrafos siguientes son enunciados.
1. Los índices de vulnerabilidad han logrado demostrar que es posible tene una unidad de análisis durante un largo de un periodo de tiempo. 
Díaz et al. (2002) exploraron la relación entre calor y mortalidad en Sevilla (España), concluyendo que el aumento de la mortalidad ocurre abruptamente, especialmente en personas mayores, a partir de 36,5 y $41^{\circ} \mathrm{C}$ de temperatura máxima; en tanto Azhar et al. (2014) estudiaron la misma relación en Gujarat (India), encontrando que la ola de calor del verano del 2010 provocó una gran cantidad de mortandad. Debido al limitado alcance geográfico y temporal los hallazgos de ambas investigaciones no son generalizables.

De manera similar, Michelozzi et al. (2005) estudiaron el impacto de la ola de calor en la mortalidad por causa específica y el papel de las características demográficas y las condiciones socioeconómicas que pueden haber aumentado el riesgo de mortalidad en cuatro ciudades italianas: Bolonia, Milán, Roma y Turín para el año 2003. Sus resultados mostraron que, los adultos mayores de las cohortes 75-84 años y 85 años y más fueron los grupos etarios más afectados; el nivel de la mortalidad era mayor entre las mujeres; el mayor incremento de la mortalidad se registró en personas con un estatus socioeconómico bajo (Roma $+17,8 \%$ ) y con niveles educativos más bajos en Turín (+ 43\%).

Ahmadalipour y Moradkhani (2018) realizaron también una medición del riesgo de mortalidad asociado con el estrés por calor excesivo en Oriente Medio y Norte África para personas mayores de 65 años, encontrando que el riesgo de mortalidad se incrementará de 8-20 en los primeros 30 años del siglo XXI con respecto al período histórico 1951-2005. Los autores sugieren que gran parte del aumento en el riesgo de mortalidad se debe al incremento en la frecuencia de los días cálidos en lugar de su intensidad.

Por otro lado, Singh y Singh (2015) examinaron las muertes por rayos en la India en el periodo de 1979 a 2011, los resultados muestran que un número significativo de hombres (89\%) ha muerto por relámpagos en comparación con mujeres $(5 \%)$ y niños $(6 \%)$, lo que es más probable debido a la mayor proporción de hombres que trabajan y se mueven al aire libre en condiciones de soledad.

Zagheni, Muttarak y Striessnig (2015) en cambio evaluaron para el período 1995-2011 en 63 países los patrones de mortalidad de los desastres hidrometeorológicos en las poblaciones a través de las dimensiones de la edad, el sexo y el desarrollo humano. Encontraron que las tasas de mortalidad por desastres hidrometeorológicos para los hombres son consistentemente más altas que para las mujeres en todos los grupos de edad, y que el diferencial por sexo es mayor para los adultos que para los niños pequeños o los ancianos.

Los estudios referidos han proporcionado información importante sobre el impacto que los eventos climáticos extremos pueden tener en la vida humana, adicionalmente se cuenta con algunos indicios que sugieren que la prevalencia de muertes en estos casos depende del tipo de desastres, del género y del nivel socioeconómico, por ejemplo en el caso de muertes asociadas a olas de calor, éstas pueden estar relacionadas con escasez de recursos económicos, tarifas dinámicas de energía eléctrica, hacinamiento y jefas de familia de edad avanzada. Con esto se da parte de que aún se carece todavía de información detallada sobre grupos vulnerables. Los diferentes periodos a estudio, espacialidades y diferencias metodológicas dificultan la comparación de resultados de estos trabajos.

Es difícil encontrar investigaciones que analicen todas las causas de muerte de todos los riesgos naturales y las características sociodemográficas durante un largo período de tiempo y con una cobertura geográfica amplia, más allá de la acumulación del número de víctimas por desastre de origen meteorológico. Aunque existen algunas excepciones, por ejemplo:

- Myung y Jang (2011) analizaron las causas específicas y las características demográficas y regionales de la muerte debido a desastres meteorológicos en Corea del Sur entre 1990 y 2008, encontraron que las inundaciones causaron el mayor número de muertes, tendencia que está cambiando a tifones. Los factores asociados con una mayor vulnerabilidad se asocian a personas residentes en provincias costeras, de edad avanzada y del sexo masculino.

- Mientras que Mahapatra, Walia, Saggurti (2018) calcularon la mortalidad relacionada con eventos climáticos extremos registrada en la India durante 2001-2014, empleando como variables analíticas la región de residencia, la edad y el sexo. La investigación concluye que la mayoría de las muertes inducidas por eventos climáticos extremos se 
debieron a rayos, seguidos de precipitaciones y temperaturas muy altas o muy bajas; hay una sobremortalidad masculina; las personas se adaptan a algunos fenómenos meteorológicos, como las olas de frío y los ciclones.

La mayoría de los estudios realizados sobre la mortalidad por peligros naturales en México, se han centrado en un tipo específico de desastre de origen meteorológico, como el calor. Dos investigaciones sobresalen en esta línea, en la primera Rojas-Rodríguez y Hurtado-Díaz (2006) elaboraron un diagnóstico sobre los efectos del cambio climático en la salud humana de la población en México y examinaron las muertes por golpe de calor durante el periodo 1979-2003, concluyendo que 1998 fue el año en donde se registraron más muertes por calor y que los estados de Sonora y Baja California eran las entidades que concentran el mayor número de muertes de este tipo.

Rojas-Rodríguez y Hurtado-Díaz (2006) señalan que el aumento en la temperatura ambiental incrementa la mortalidad por golpe de calor y una mayor incidencia en casos de dengue, paludismo y enfermedades diarreicas, además de una mayor mortalidad por enfermedades respiratorias relacionada con el aumento en la temperatura ambiente y la contaminación atmosférica.

En la segunda investigación, Díaz, Castro y Aranda (2014) analizaron el impacto de las altas temperaturas en la mortalidad durante el período 2002-2010 e identificaron 393 decesos de personas por calor natural excesivo, la mayor parte ubicados en el noroeste de México, hombres con un bajo nivel socioeconómico.

Con base en el contexto presentado es pertinente examinar los cambios en la mortalidad por desastres meteorológicos, así como analizar las características y las diferencias regionales de las personas que murieron según los tipos de desastre ocurridos durante el periodo 2000-2015 en México.

\section{METODOLOGÍA}

En México existen diferentes tipos de registros administrativos sobre estadísticas de diversa índole como, seguridad pública y justicia (impartición de justicia en materia penal y organismos públicos de derechos humanos); económicas (accidentes de tránsito terrestre en zonas urbanas y suburbanas, balanza comercial de mercancías de México, exportaciones por entidad federativa, industria minero metalúrgica, perfil de las empresas manufactureras de exportación, programa de la industria manufacturera, maquiladora y de servicios de exportación (IMMEX), sacrificio de ganado en rastros municipales, transporte urbano de pasajeros y vehículos de motor registrados en circulación); sociales (museos, relaciones laborales de jurisdicción local y salud en establecimientos particulares); históricas (cultura, industria maquiladora de exportación, intentos de suicidio, suicidios y judiciales en materia penal); y hechos vitales (natalidad, nupcialidad y mortalidad), los cuales se actualizan de manera periódica (INEGI, 2019).

Entre los registros administrativos mencionados el de hechos vitales y en particular las estadísticas de mortalidad fueron fundamentales para el desarrollo de esta investigación al tratarse de la fuente de información primaria. Las estadísticas sobre defunciones son contabilizadas en México desde 1893 a la fecha, aunque se tiene datos disponibles en formato de base de datos desde 1985 (INEGI, 2018)

Las entidades gubernamentales encargadas de sistematizar y elaborar las estadísticas sobre defunciones en México son la Secretaría de Salud y del Instituto Nacional de Geografía e Informática (SSA-INEGI), a quienes por ley cada vez que ocurre un deceso el registro civil les envía copia del certificado defunción, para que capturen la información de la persona fallecida.

En el certificado de defunción es un requisito indispensable para poder enterrar o cremar a una persona y debe de ser llenado por algún médico quien debe anotar en la causa especifica que originó la muerte y algunas características de la persona fallecida, cuando se vive en un medio rural aislado es la autoridad principal de la localidad la encargada de realizar esta tarea. Es llenado siguiendo los lineamientos de la Organización Mundial de la Salud señalados en la Clasificación Estadística Internacional de Enfermedades y Problemas Relacionados con la Salud (OPS/OMS, 2019) para identificar la causa específica de la defunción y tener comparabilidad internacional.

Además, en el certificado de defunción son captadas de manera adicional más de 50 variables sobre el espacio geográfico de residencia y características sociodemográficas y contextuales del fallecido como: entidad y municipio de registro, entidad, municipio y localidad de residencia habitual; entidad, municipio y localidad de ocurrencia de la defunción; sexo, edad, ocupación, escolaridad, estado conyugal, entre otras (SSA, 2019). 
La Clasificación Estadística Internacional de Enfermedades y Problemas Relacionados con la Salud (CIE) identifica en su décima edición entre las distintas causas de defunción las ocasionadas por exposición a fuerzas de la naturaleza que son (OPS, 2018):

- Exposición al calor natural excesivo

- Exposición al frío natural excesivo

- Exposición a rayos solares

- Víctima de rayo

- Víctima de terremoto

- Víctima de erupción volcánica

- Víctima de avalancha, derrumbe y otros movimientos de tierra

- Víctima de tormenta cataclísmica

- Víctima de inundación

- Exposición a otras fuerzas de la naturaleza, y las no especificadas

El objetivo de esta investigación es conocer el estado actual y los cambios en la mortalidad por exposición a fenómenos de la naturaleza durante el periodo 2000-2015, además de identificar si hay un perfil entre las personas que murieron por estas causas. Para ello se emplearon los microdatos del registro de defunciones para el periodo señalado.

Los datos sobre las muertes por desastre de origen meteorológico durante el periodo 2000$2015(t)$ son presentados en la investigación en números absolutos y en tasas para resaltar los riesgos a los que se enfrenta la población en México. Las tasas se expresan por millón de personas y se calcula:

\begin{tabular}{|c|c|c|}
\hline \multirow{2}{*}{$\begin{array}{c}\text { TMDM } \\
\text { Tasa de Muertes por Desastre de } \\
\text { Origen Meteorológico }\end{array}$} & $\begin{array}{l}\mathrm{N}^{0} \text { de defunciones ocurridas por desastre } \\
\text { meteorológicos durante el periodo } t\end{array}$ & \multirow{2}{*}{$\times 1.000 .000$} \\
\hline & $\begin{array}{c}N^{0} \text { de personas expuestas al riesgo durante el } \\
\text { periodo } t\end{array}$ & \\
\hline
\end{tabular}

Los microdatos de la mortalidad en México de los cuales fueron seleccionadas las muertes por desastre de origen meteorológicos, se procesaron en el programa Stata V.11, los gráficos se realizaron en MS Excel y los mapas en MapViewer.

\section{RESULTADOS}

Durante el periodo 2000-2015 ocurrieron en México 4.177 muertes por desastres meteorológicos, siendo el año 2009 la cohorte de tiempo en que se registró la cifra más baja 203 muertes y el año 2004 la más alta con 324 muertes. Del total de muertes poco más de un tercio 1.424 se registraron entre los años 2010 y 2015.

Analizando la incidencia de mortalidad por desastres meteorológicos en México según año de ocurrencia, se aprecia a lo largo del periodo estudiado una ligera tendencia a la baja (ver Figura 1), pero se mantiene constante la letalidad de los eventos climáticos extremos con una tasa cercana a 2 defunciones por cada millón de personas anuales en promedio, en el año 2015 la tasa fue de 2,1. Las tasas para México presentan una tendencia diferente a la sugerida por Goklany (2007) quien señala una clara tendencia a la baja en el mundo de la mortalidad producida por los desastres meteorológicos².

Por causa específica de muertes según tipo de evento meteorológico extremo ocurrido en el periodo de estudio (ver Figura 2), destaca que la mayoría de las muertes por desastres meteorológicos se debieron a rayos $(46,8 \%)$, le siguieron por frío excesivo $(20,2 \%)$, por golpe de calor $(17,8 \%)$, avalanchas o derrumbes $(7,6 \%)$, tormenta $(2,4 \%)$, inundación $(1,8 \%)$, exposición a rayos solares $(1,7 \%)$, terremoto $(1,0 \%)$, erupción volcánica $(0,1 \%)$ y otras $(0,7 \%)$.
2. Aunque podría ser que las

variaciones de los últimos tres años se deban a fluctuaciones aleatorias por lo que se es necesario aplicar un análisis estadístico para confirmar que no hay un descenso de la tasa de defunciones. 


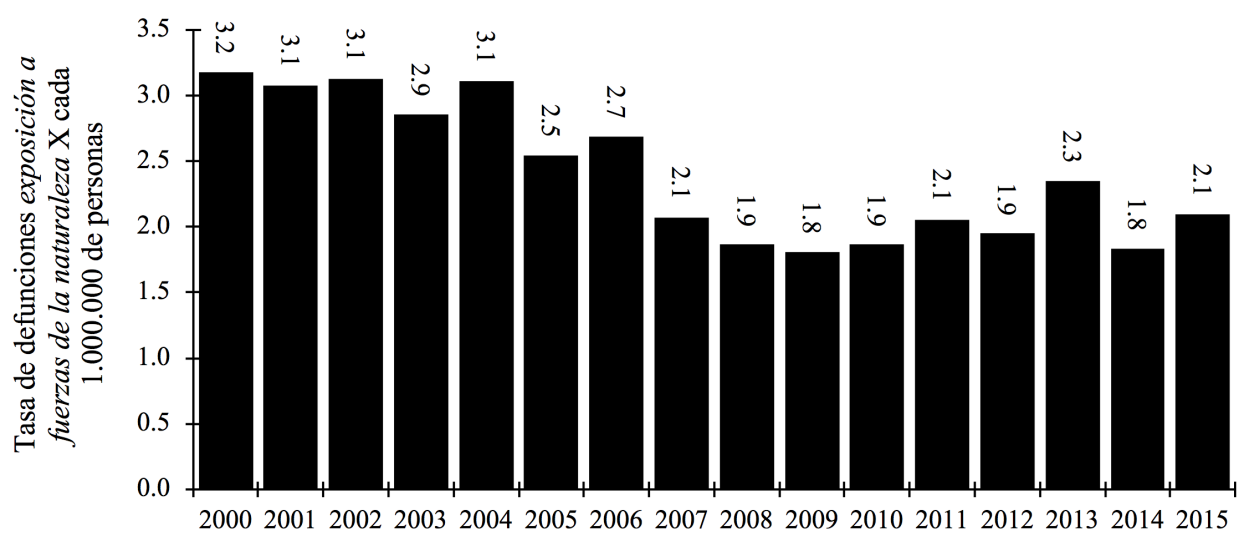

Año

Figura 1. Tasas de defunciones por exposición a desastres meteorológicos en México, durante los años 2000 a 2015

Fuente: Elaboración propia con base en los microdatos de defunción en México, INEGI-SSA, 2000-2015.

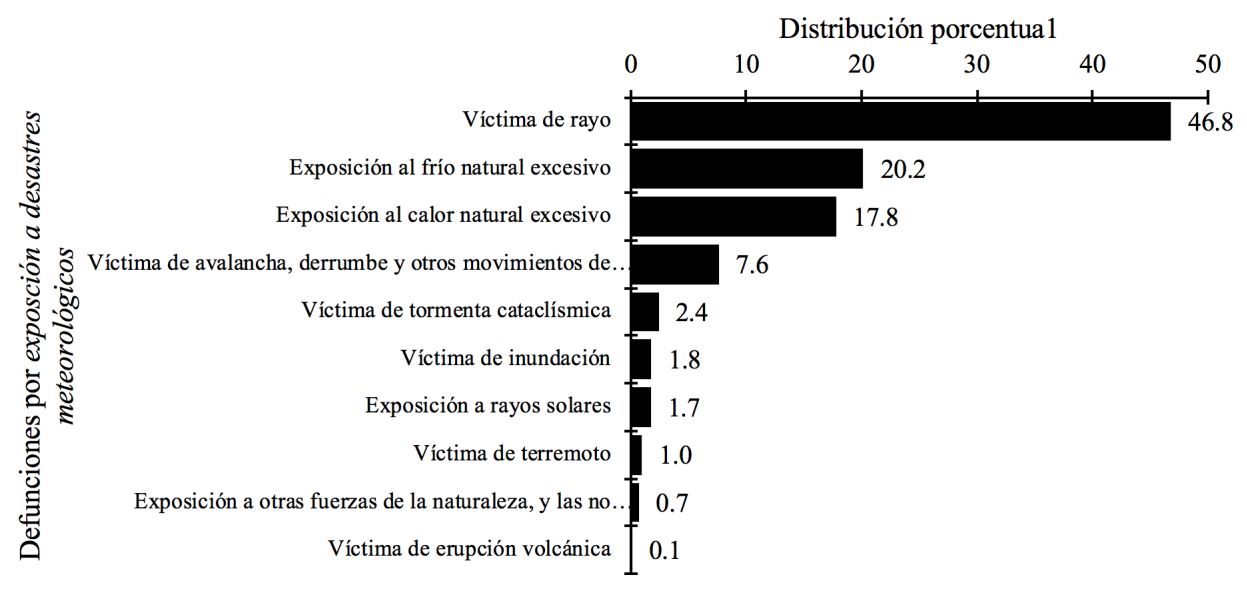

Figura 2. Distribución porcentual de muertes por desastres meteorológicos en México según causas, 2000 a 2015 Fuente: Elaboración propia con base en los microdatos de defunción en México, INEGI-SSA, 2000-2015.

Los desastres meteorológicos a menudo catastróficos y altamente destructivos como los huracanes y terremotos provocaron menos muertes en comparación con otros desastres meteorológicos menos catastróficos como, los rayos, la exposición al frío extremo o el golpe de calor.

En la evolución de las muertes por causa específica y tipo de evento meteorológico se observa que cada uno sigue una tendencia diferente (ver Figura 3). Entre 2000 y 2015, las muertes por los golpes de calor, avalanchas, tormentas cataclísmicas, inundaciones y terremotos se han incrementado mientras que las muertes por olas de frío y rayo han disminuido.

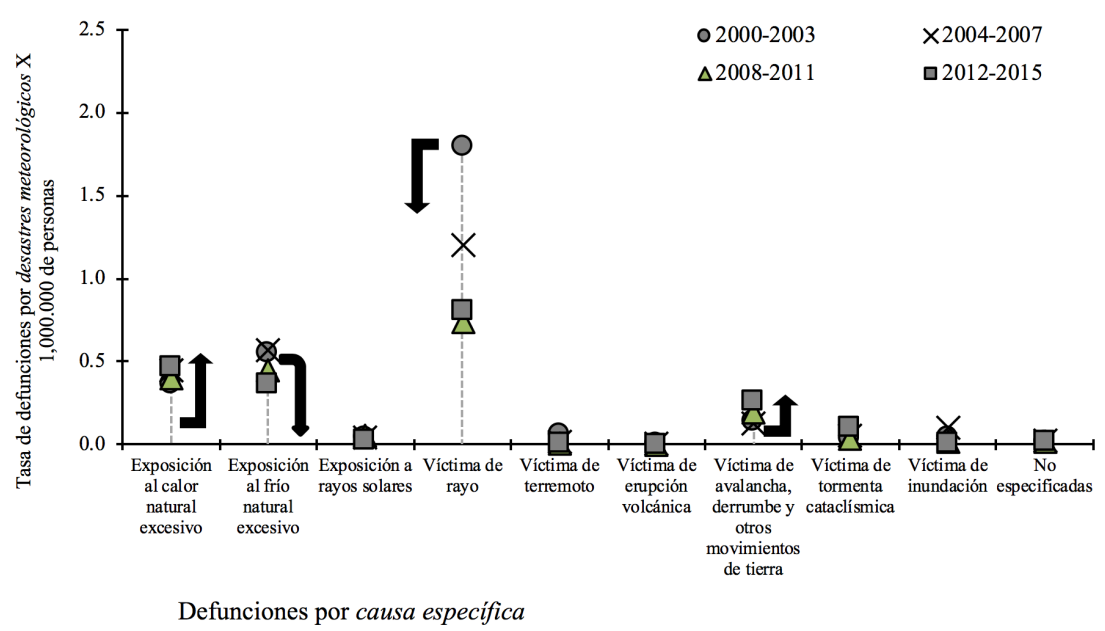

Figura 3. TMDMs en México según causas, en cuatro cohortes de tiempo durante el período 2000-2015

Fuente: Elaboración propia con base en los microdatos de defunción en México, INEGI-SSA, 2000-2015. 
Según espacio geográfico de residencia la tasa acumulada de muertes por eventos meteorológicos 2000-2015 presentan variaciones (ver Figura 4). Los estados de Sonora (9,9 muertes por un millón), Chihuahua (7,5), Zacatecas (4,6), Guerrero $(3,8)$, Michoacán $(3,4)$, Nayarit $(3,5)$, Oaxaca $(3,6)$, San Luis Potosí $(3,2)$ y Coahuila $(3,1)$ son los que registraron las tasas más altas, las cuales fueron superiores a las estimada a nivel mundial por Goklany, en el año 2007, que fue de 3 muertes por millón.

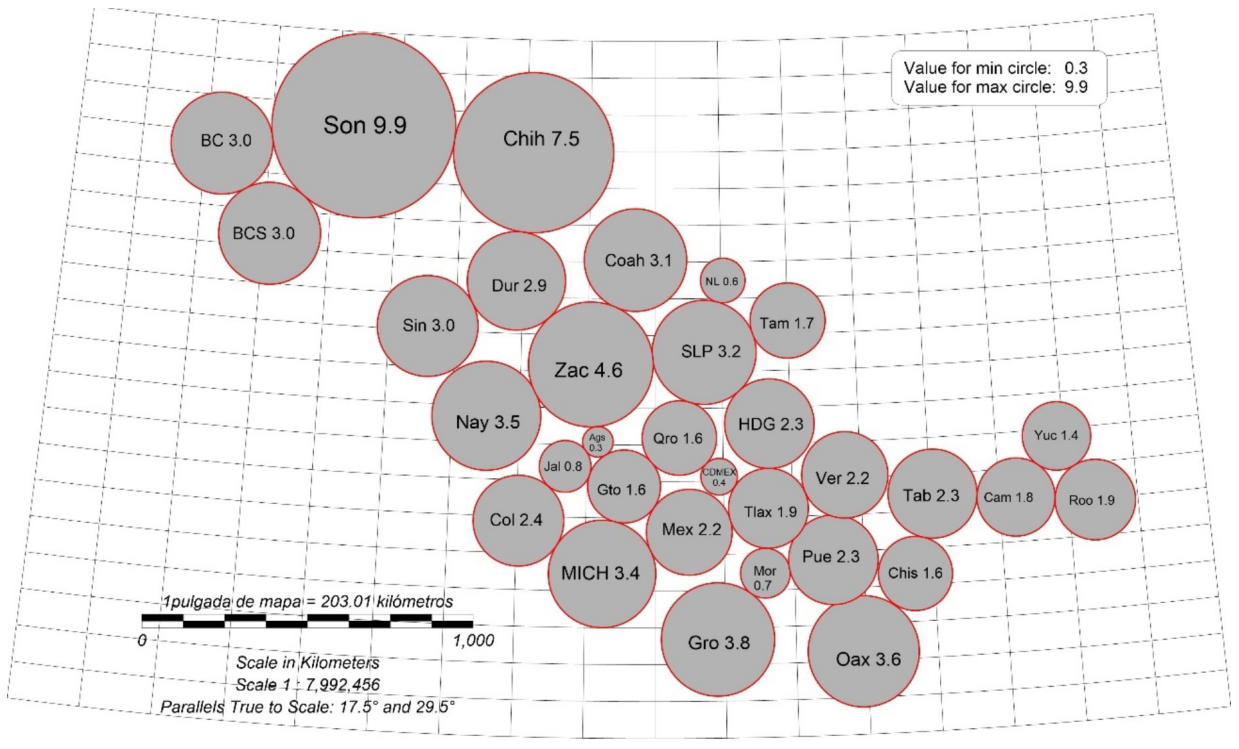

Figura 4. TMDM acumuladas en México según entidad de ocurrencia para el periodo 2000-2015 Fuente: Elaboración propia con base en los microdatos de defunción en México, INEGI-SSA, 2000-2015.

La comparación de la distribución porcentual de las muertes según el tipo de desastre de origen meteorológico en cinco tamaños de localidades mostró diferencias en el nivel de incidencia (ver Figura 4). Por ejemplo, las muertes por rayo tienen un mayor peso porcentual en las localidades de menor tamaño (menos de 15.000 habitantes), donde se concentraron más del 50\%, mientras en las ciudades metropolitanas (localidades de más de quinientos mil habitantes) solo ocurrió $8,5 \%$ de este tipo de muertes. En general, a medida que disminuye el tamaño de la localidad de ocurrencia aumenta la incidencia de muertes por rayo.

Las muertes por los golpes de frío o calor tienen en cambio una concentración mayor en las localidades más grandes, en las ciudades metropolitanas (localidades de más de quinientos mil habitantes) del total de las muertes por exposición a fenómenos de la naturaleza, 41,8\% fueron por frío extremo mientras que $38,2 \%$ fue por golpe de calor. En contraste, en localidades rurales las muertes por frío extremo representaron $15,5 \%$ y por golpe de calor $9,4 \%$ del total, respectivamente.

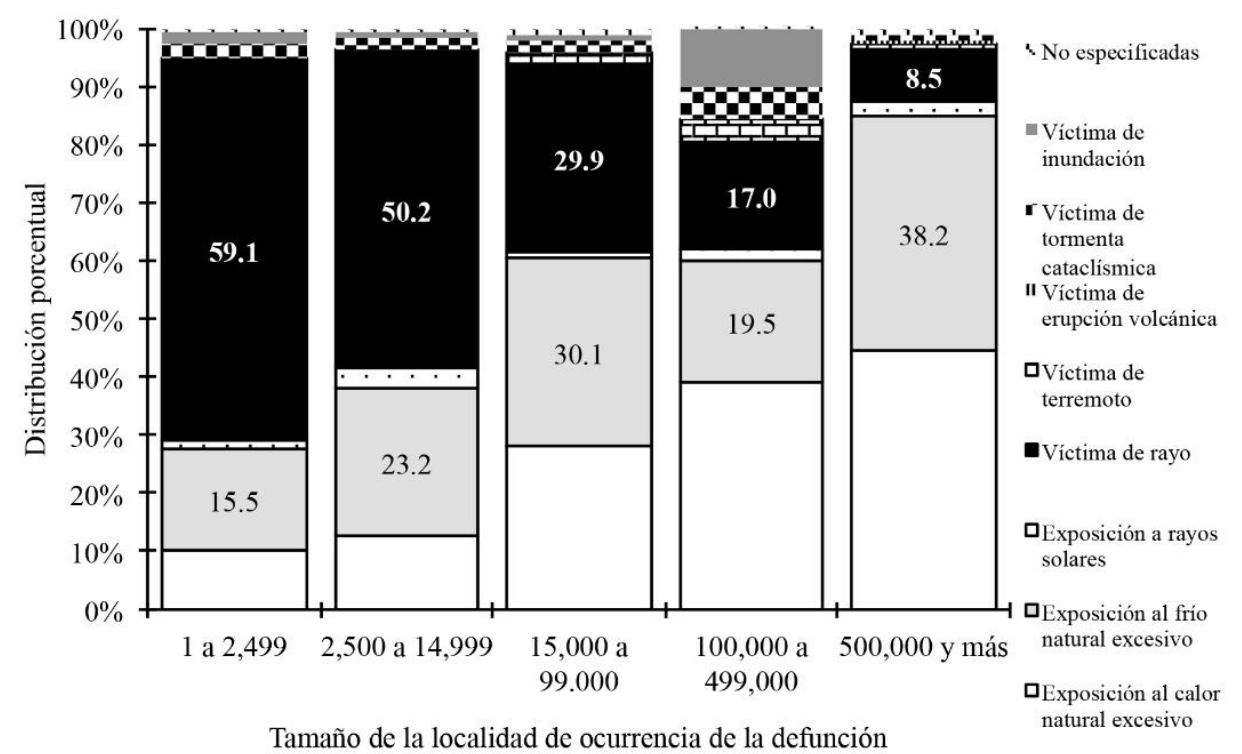

Figura 5. Distribución porcentual de muertes por desastres meteorológicos en México según tamaño de localidad de 
Mientras que las muertes por exposición a los rayos del sol no siguen un patrón definido, ocurren en una proporción similar en todos los tamaños de localidades. En cambio, las muertes por tormenta, inundación y terremoto en mayor proporción ocurren en ciudades intermedias de 100 mil a 499 mil habitantes.

Comparando entre sexos el total acumulado de muertes por desastres meteorológicos, $81.2 \%$ fueron hombres y $18,8 \%$ mujeres. Por tamaño de la localidad de ocurrencia la diferencia por sexo se mantiene en los cinco tamaños analizados: 1 a 2.499; 2.500 a 14.999; 15.000 a 499.000 y 500.000 y más.

Según el tipo de desastre se aprecian diferencias importantes en la composición por sexo (ver Figura 6), más hombres murieron por olas de frío $(88,6 \%)$, golpe de calor $(81,6 \%)$, rayo $(80,6 \%)$, avalancha $(80,5 \%)$ y rayos solares $(80,3 \%)$. Mientras que más mujeres murieron por erupción volcánica $(66,7 \%)$, inundación $(45,9 \%)$, terremoto $(36,6 \%)$ y tormenta cataclísmica $(35,0 \%)$. Hay una clara relación entre la división del trabajo por género con el riesgo de morir por un desastre específico.

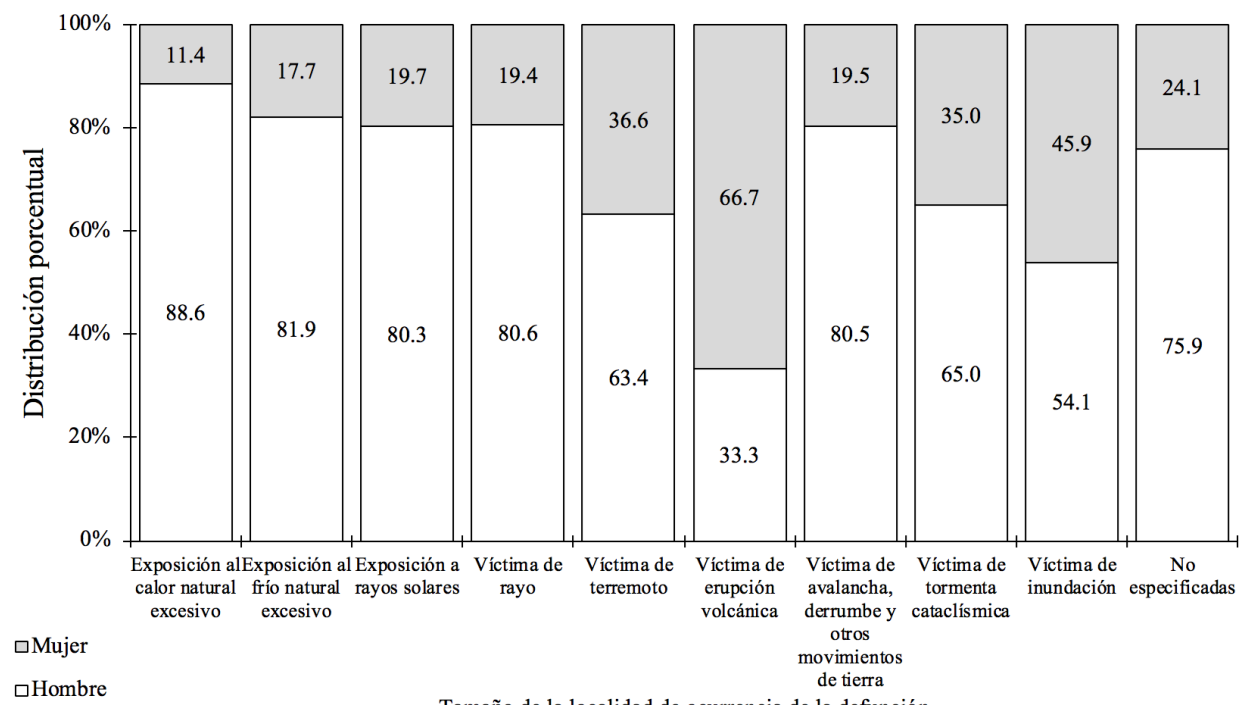

Figura 6. Distribución porcentual de muertes por desastres meteorológicos en México según sexo y tipo, 2000 a 2015 Fuente: Elaboración propia con base en los microdatos de defunción en México, INEGI-SSA, 2000-2015.

Las mujeres presentan un mayor riesgo de morir por desastres meteorológicos catastróficos altamente destructivos, por colapso infraestructural, en particular de las viviendas ${ }^{3}$, mientras que los hombres tuvieron un mayor riesgo de morir por un evento meteorológico no catastrófico o menos destructivos que regularmente ocurren en espacios abier tos ${ }^{4}$. Estos resultados concuerdan con los hallazgos señalados por de Singh y Singh (2015).

Los hombres mueren más que las mujeres por desastres meteorológicos por imprudencia, al creer que no necesitan tomar precauciones son más propensos asumir riesgos sin evaluar la gravedad de la situación a la que se enfrentan como golpes de frío o calor extremo.

Las víctimas por desastre de origen meteorológico tenían al momento de su muerte en promedio 40 años, diferenciando por edad y sexo los hombres presentan una mayor edad promedio de 40,9 años en comparación con las mujeres de 38,4 años. Las diferencias por sexo se agudizan en la distribución de la edad según percentiles, ya que en el primer cuartil (o el percentil 25) las mujeres apenas alcanzan los 15 años y los hombres los 24 años, esto muestra que entre las mujeres las niñas tienen un mayor riesgo de morir por un desastre de origen meteorológico a diferencia de los hombres.

Según el tipo de evento meteorológico se observaron diferencias en la edad promedio, siendo víctimas por erupción volcánica las de mayor edad al momento de la defunción con una media de 78 años, le siguen los que murieron por, rayos solares con una media de 53 años y frío extremo con 52 años promedio. En tanto, las víctimas con menor edad entre 32 y 35 años en promedio murieron por avalancha, rayo y tormentas.
3. Gasman (2019) señala que: "La Ciudad de México ha sido testigo de la vulnerabilidad de las mujeres y niñas a los riesgos de desastres, como el vivido el pasado 19 de septiembre de 2017, donde las cifras oficiales reflejaron que, de un total de 228 per fallecidas y rescatadas posteriormente en edificios colapsados en la Ciudad, 138 fueron mujeres; es decir, dos mujeres por cada hombre" (p. 1).

4. Los hombres tienen una mayor participación laboral en actividades como la agricultura, la construcción u otras al aire libre, esto aumenta su exposición a desastres meteorológicos como rayos, avalanchas y rayos solares. 
En la distribución por percentiles, sobresale que las víctimas por inundaciones y tormenta en comparación con los otros desastres meteorológicos afecta en mayor medida a personas de menor edad, principalmente niños y niñas, en el primer cuartil las víctimas de estos eventos apenas alcanzaron la edad de 11 y 14 años, respectivamente. En contraste eventos como erupción volcánica, frío excesivo y terremoto afectaron principalmente a los adultos mayores, en el tercer cuartil (o percentil 75) las víctimas de estos desastres tenían en promedio 83, 75 y 71 años.

En la pirámide de edad y sexo de las muertes por desastres meteorológicos para el periodo 2000-2015 sobresale las diferencias entre hombres y mujeres que le dan una forma atípica. Así las defunciones de hombres se concentran en las edades productivas y en el grupo de adultos mayores de 65 años y más, en cambio entre las mujeres las defunciones permanecen en un nivel similar en todos los grupos etarios, para tener un crecimiento exponencial en la cohorte de 65 años y más (ver Figura 7).

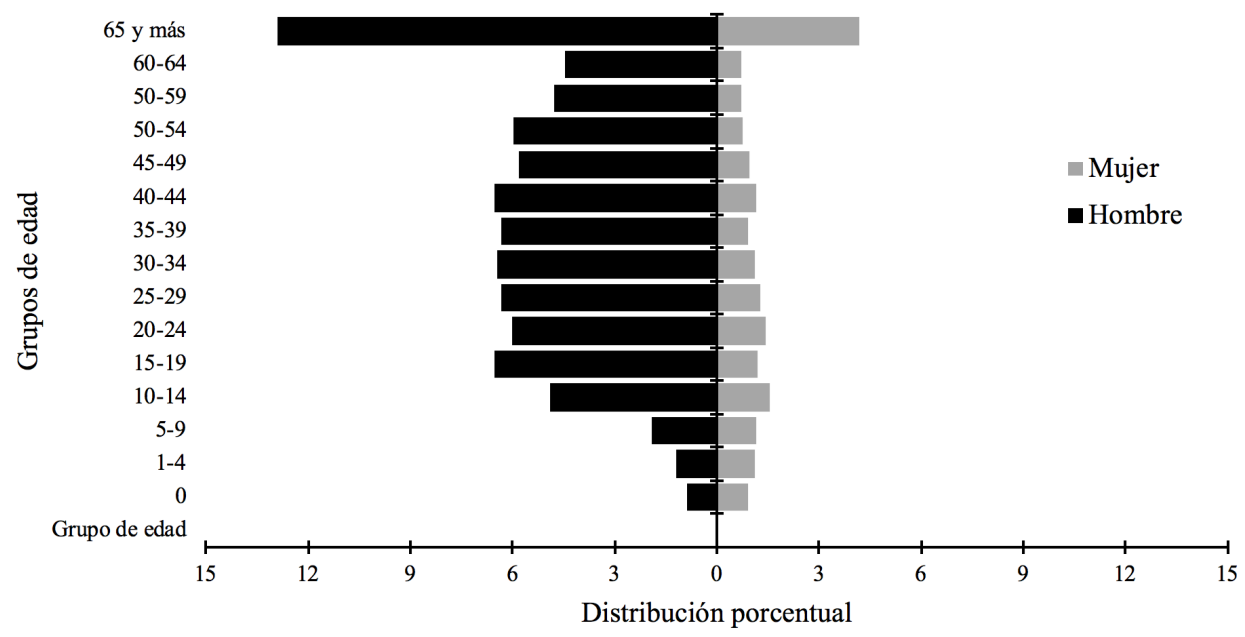

Figura 7. Distribución porcentual de muertes por desastres meteorológicos en México según grupo de edad y sexo para el periodo 2000-2015

Fuente: Elaboración propia con base en los microdatos de defunción en México, INEGI-SSA, 2000-2015.

Las diferencias en la composición por sexo y edad de la población que ha sido víctima de un evento meteorológico cambian de acuerdo con el tipo de desastre al que estuvieron expuestos, en la Figura 8 se muestran las pirámides para cuatro tipos de desastres meteorológicos: golpe de calor, frío extremo, rayo y avalancha, que son los de mayor relevancia numérica.

Para el caso del calor extremo, en los hombres las muertes se concentraron en las cohortes de edad de 20 a 54 años y en los de 65 años y más, mientras que en las mujeres fueron en la de 65 años y más. En tanto, las víctimas de golpe de frío en la pirámide las muertes se centralizan en los hombres de 45 a 49 años junto con los mayores de 65 años, entre las mujeres la cohorte etaria más afectada por tipo de evento fueron también adultas mayores de 65 años y más y niños(as) de 0 a 5 años (ver Figura 8).

Las muertes por rayo, en los hombres se concentran en la cohorte etaria de 10 a 19 años y en las mujeres en el grupo de 10 a 14 años. Mientras que en las muertes por avalanchas los más afectados fueron hombres de las cohortes etarias de 15 a 39 años y las mujeres de dos cohortes niñas de 1 a 9 años y jóvenes de 20 a 29 años (ver Figura 8).

El riesgo de morir por un desastre de origen meteorológico es diferente según el nivel de escolaridad, $43 \%$ de las personas que murieron por esta causa no tenían ningún grado de instrucción, 19,5\% había estudiado algún grado de educación primaria; en tanto, 15,6\% de las víctimas poseía un nivel de estudios básicos (primaria y secundaria), 4,8\% estudios intermedios (bachillerato) y solo 10,3\% contaba con estudios universitarios (ver Figura 9).

Comparando por sexo, las mujeres víctimas de algún desastre de origen meteorológico tenían una menor escolaridad en comparación con los hombres. Del total de víctimas del sexo femenino $41,5 \%$ no tenían ningún nivel instrucción proporción que entre los hombres desciende hasta el $33,9 \%$. Las mujeres que murieron por un evento meteorológico extremo tienen una doble condición de vulnerabilidad, ser mujeres y analfabetas (ver Figura 9). 

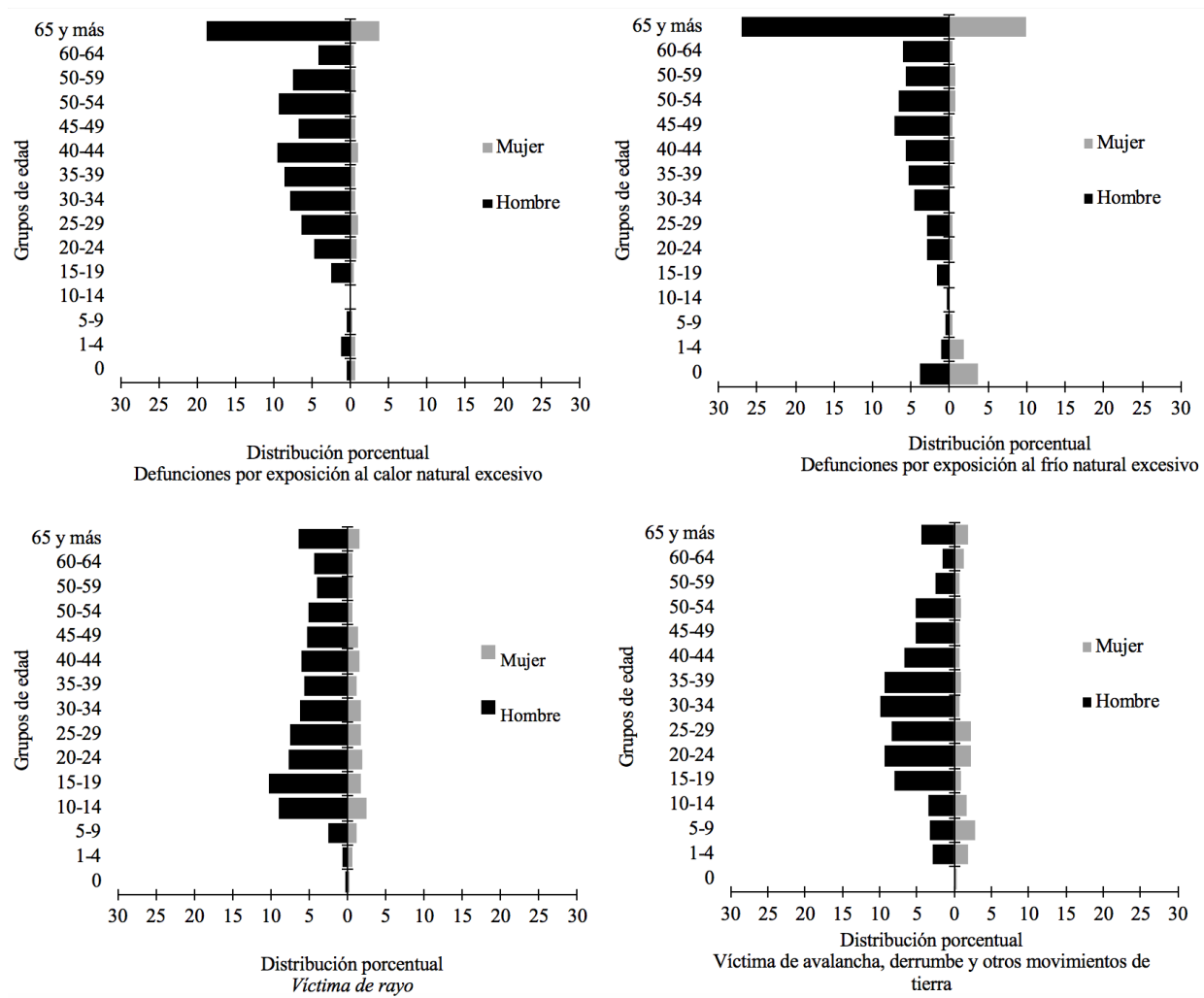

Figura 8. Distribución porcentual de muertes por desastres meteorológicos en México por grupo de edad, sexo y causa específica, para el periodo $2000-2015$

Fuente: Elaboración propia con base en los microdatos de defunción en México, INEGI-SSA, 2000-2015.

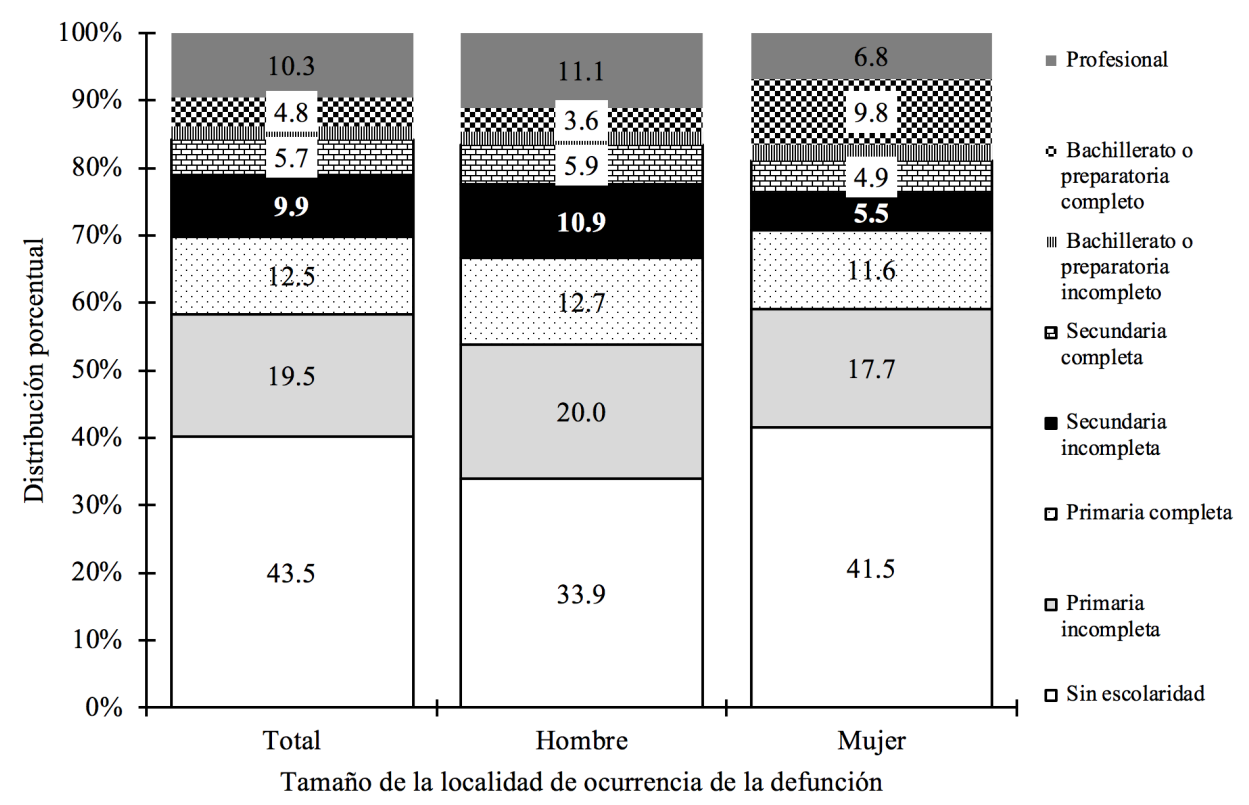

Figura 9. Distribución porcentual de muertes por desastres meteorológicos en México, por escolaridad y sexo para el periodo 2000-2015

Fuente: Elaboración propia con base en los microdatos de defunción en México, INEGI-SSA, 2000-2015.

Diferenciado el nivel de la escolaridad de las víctimas por causa especifica de mortalidad de las víctimas por evento meteorológico, se observó que el nivel educativo tiene un efecto diferencial en el riesgo de morir por un tipo de desastre meteorológico específico, por ejemplo, una mayor proporción de personas sin escolaridad murieron por una erupción volcánica, rayos solares y golpe de frío, en contraparte al tener mayor escolaridad es más probable ser víctimas de un terremoto, frío excesivo y golpe de calor (ver Tabla 1). Se confirma que la falta de educación formal puede contribuir a incrementar el riesgo ante ciertos eventos climáticos extremos. 
En cuanto a la ocupación de las personas que fueron víctimas de un evento meteorológico $29,7 \%$ no trabajaba. Entre quienes realizaban una actividad económica, resalta que $44,5 \%$ trabajaba en actividades agrícolas, ganaderas, forestales, caza y pesca, 9,1\% estaba ocupado en la industria de la transformación ${ }^{5}$ y 3,2\% eran personal administrativo de nivel inferior. En conjunto efectúan actividades económicas que requiere un nivel de calificación y salario bajo, se corrobora que las personas pobres tienen una mayor exposición al riesgo de morir por un desastre de origen meteorológico

\begin{tabular}{lllll} 
Causa & Sin & $\begin{array}{l}\text { Educación } \\
\text { escolaridad }\end{array}$ & $\begin{array}{l}\text { Educación } \\
\text { Báca }\end{array}$ & $\begin{array}{c}\text { Educación } \\
\text { Superior }\end{array}$ \\
\hline Exposición al calor natural excesivo & 30.6 & 46.2 & 7.1 & 16.1 \\
\hline Exposición al frío natural excesivo & 41.8 & 29.9 & 9.9 & 18.5 \\
\hline Exposición a rayos solares & 45.5 & 44.0 & 3.0 & 7.6 \\
\hline Víctima de rayo & 35.5 & 55.0 & 4.3 & 5.3 \\
\hline Víctima de terremoto & 33.3 & 33.4 & 12.9 & 20.5 \\
\hline Víctima de erupción volcánica & 66.7 & 33.3 & 0.0 & 0.0 \\
\hline $\begin{array}{l}\text { Víctima de avalancha, derrumbe y otros } \\
\text { movimientos de tierra }\end{array}$ & 27.4 & 55.1 & 9.6 & 7.9 \\
\hline $\begin{array}{l}\text { Víctima de tormenta cataclísmica } \\
\text { Víctima de inundación }\end{array}$ & 36.3 & 44 & 13.2 & 6.6 \\
\hline $\begin{array}{l}\text { Exposición a otras fuerzas de la naturaleza, y las no } \\
\text { especificadas }\end{array}$ & 21.4 & 43.5 & 13.7 & 11 \\
\hline
\end{tabular}

Tabla l. Distribución porcentual de muertes por desastres meteorológicos en México, por escolaridad y causa específica para el periodo 2000-2015

Fuente: Elaboración propia con base en los microdatos de defunción en México, INEGI-SSA, 2000-2015.

En el caso particular de las personas que desempeñaban actividades agrícolas, ganaderas, forestales, caza y pesca en su mayoría $64,0 \%$ murieron por rayo; $13,8 \%$ por frío extremo y $13,2 \%$ por calor excesivo. Los datos muestran que los trabajadores agrícolas pudieron tener menor conocimiento y capacidad para adaptarse a los eventos meteorológicos no catastróficos, por lo tanto, la información sobre cómo afrontar las condiciones meteorológicas de las tormentas eléctricas y la temperatura deben ser priorizadas para los trabajadores que desarrollan actividades agrícolas.

\section{REFLEXIONES FINALES}

En esta investigación ha demostrado que los impactos de los desastres no se distribuyen uniformemente entre los subgrupos de población, debido a que la vulnerabilidad es multidimensional y dinámica. Identificar quién es vulnerable a qué peligro es fundamental en los esfuerzos de intervención para reducir la vulnerabilidad.

La mortalidad por desastres ocasionada por eventos climáticos extremos continúa siendo inaceptablemente alta, ya que la mayoría de esas muertes podrían haberse evitado. La letalidad de los desastres se produce no solo por la exposición a una determinada amenaza, sino también, por la vulnerabilidad acumulada en que se encuentran determinadas poblaciones.

Teniendo en cuenta los resultados de la investigación, los programas de prevención deben dirigirse específicamente a los hombres en edades productivas y adultos mayores, a las mujeres en particular a las niñas menores de nueve años y adultas mayores, lo que reduciría el impacto sobre la mortalidad por eventos meteorológicos.

Destaca entre los hallazgos que la letalidad de la exposición prolongada al frio o calor afecta en mayor medida a los hombres en edad adulta que representan $46 \%$ del total de las muertes por eventos meteorológicos extremos durante el periodo 2000-2015, situación que se corrige incrementando los niveles de educación, como lo muestra la misma investigación.

Esto es relevante debido a que las proyecciones del cambio climático para México muestran que a lo largo del próximo siglo las olas de calor y frío serán más frecuentes, intensas y durarán más tiempo, incluso en regiones en las que no se caracterizan actualmente por olas de calor o frío, por lo que se necesita desarrollar programas para reducir la letalidad de estos desastres de origen meteorológicos.

5. Los trabajadores considerados en este grupo realizan sus actividades en un taller, local comercial, en minas o canteras, en obras de construcción, en la vía pública, en su propio domicilio o en el domicilio del cliente. 
Para reducir el riesgo de los desastres es necesario incrementar la seguridad y la resiliencia de las comunidades. Esta estrategia forma parte del Objetivo 13: Acción Climático de los Objetivos del Desarrollo Sostenible.

\section{REFERENCIAS}

Ahmadalipour, A. \& Moradkhani, H. (2018). Escalating heat-stress mortality risk due to global warming in the Middle East and North Africa (MENA). Environment International, 117, 215-225.

Álvarez, I. \& Cadena, E. (2006). Índice de Vulnerabilidad Social en los Países de la OCDE. (U. A. Madrid, Ed.) Economic Analysis Working Paper Series. Madrid: OCDE.

Arcos González, P. I., Castro Delgado, R., \& Busto Prado, F. D. (2002). Desastres y salud pública: un abordaje desde el marco teórico de la epidemiología. Revista Española de Salud Pública, 76, 121-132.

Azhar, G., Mavalankar, D., Nori-Sarma, A., Rajiva, A., Dutta, P., Jaiswal, A., Sheffield, P., Knowlton K. \& Hess, J. (2014). Heat-related mortality in India: excess all-cause mortality associated with the 2010 Ahmedabad heat wave. PLoS One, 9(3), 234-246.

Bradshaw, S. \& Arenas, A. (2004). Análisis de género en la evaluación de los efectos socioeconómicos de los desastres naturales. Santiago de Chile: CEPAL.

Chau, P. H., Gusmano, M. K., Cheng, J. O., Cheung, S. H. \& Woo, J. (2014). Social vulnerability index for the older people-Hong Kong and New York city as examples. Journal of Urban Health, 91(6), 1048-1064.

Chaux, W. (1998). Auge, Caída y Levantada de Felipe Pinillo, Mecánico y Soldador o Yo voy a correr el riesgo. Perú: Red de Estudios Sociales en Prevención de Desastres en América Latina.

Cutter, S. L., Boruff, B. J. \& Shirley, W. L. (2003). Social Vulnerability to Environmental. Social Science Quarterly, 84(2), 242-261.

Díaz, J., García, R., Velázquez de Castro, F., Hernández, E., López, C. \& Otero, A. (2002). Effects of extremely hot days on people older than 65 years in Seville (Spain) from 1986 to 1997. International Journal Biometeorol, 46(3), 145-149.

Díaz, R., Castro, A. \& Aranda, P. (2014). Mortalidad por calor natural excesivo en el noroeste de México: Condicionantes sociales asociados a esta causa de muerte. Frontera Norte, 26(52), 155-177.

EIRD. (2007). Reducción de desastres en América Latina. Revista para las Américas, 14, 47-54.

Emanuel, K. (2005). Increasing destructiveness of tropical cyclones over the past zoyears. Nature (436), 686-688.

Flanagan, B. E., Gregory, E. W., Hallisey, E. J., Heitgerd, J. L. \& Lewis, B. (2011). A social vulnerability index for disaster management. Journal of homeland security and emergency management, 8(1), 1-22.

Gasman, N. (o4 del o6 de 2019). Desastres naturales incrementan la vulnerabilidad de las mujeres. Recuperado de: https://www.gob.mx/inmujeres/prensa/desastres-naturales-incrementan-lavulnerabilidad-de-las-mujeres [visitado el 12 de oct. 2019]

Goklany, I.M. (2009). Deaths and Death Rates from Extreme Weather Events: 1900-2008. Journal of American Physicians and Surgeons, 14(4), 102-109.

INEGI. (12 de 05 de 2019). Instituto Nacional de Geografía y Estadística. Recuperado de: https://www. inegi.org.mx/datos/?init=2\&p=regAdm [visitado el 12 de oct. 2019]

INEGI. (15 de o9 de 2018). Instituto Nacional de Geografía y Estadística. Recuperado de: https://www. inegi.org.mx/programas/mortalidad/ [visitado el 12 de oct. 2019]

INE-SEMARNAT. (2006). México Tercera Comunicación Nacional ante la Convención Marco de las Naciones Unidas sobre el Cambio Climático. Ciudad de México: Instituto Nacional de EcologíaSecretaría de Medio Ambiente y Recursos Naturales, Programa de las Naciones Unidas para el Desarrollo México, Environmental Protection Agency \& Global Environment Facility.

Kahn, M.E. (2005). The death toll from natural disasters: the role of income, geography, and institutions. Review of economics and statistics, 87(2), 271-284.

Kienberger, S., Blaschke, T. \& Zaidi, R. Z. (2013). A framework for spatio-temporal scales and concepts from different disciplines: the 'vulnerability cube'. Natural Hazards, 68(3), 1343-1369.

Kovats, R., Campbell-Lendrum, D. \& Matthies F. (2005). Climate change and human health: estimating avoidable deaths and disease. Risk Analysis, 25(6), 409-1418.

Lavell, A. (1993). Ciencias Sociales y Desastres en América Latina: Un encuentro inconcluso. Revista EURE, 21(58), 73-84. 
Lutz, W., Butz, W., Castro, M., Dasgupta, P., Demeny, P., Ehrlich, I. Giorguli, S. Habte, D., Haug, W., Hayes, A., Herrmann, M., Jiang, L., King, D., Kotte, D., Lees, M., Makinwa-Adebusoye, P., McGranahan, G., Mishra, V., Montgomery, M., Riahi, K., et al., (2012). Demography's role in sustainable development. Science, 335(6071), 918-918.

Lutz, W. \& Shah, M. (2002). Population should be on the Johannesburg agenda. Nature, 418(6893), $17-17$.

Medrano, J., y García, A. (2014). Climatología de tornados en México. Investigaciones Geográficas, Boletín del Instituto de Geografía (83), 74-87.

Magrin, G., Gay García, C., Cruz Choque, D., Giménez, J., Moreno, A., Nagy, G., Nobre, C. \& Villamizar, A. (2007) Latin America. En Climate Change 2007: Impacts, Adaptation and Vulnerability. Contribution of Working Group II to the Fourth Assessment Report of the Intergovermental Panel on Climate Change (pp. 581-615). Cambridge, UK: Cambridge University Press.

Mahapatra, B., Walia, M. \& Saggurti, N. (2018). Extreme weather events induced deaths in India 2001-2014: Trends and differentials by region, sex and age group. Weather and Climate Extremes, 21(9), 110-116.

McCarthy, J.P. (2001). Ecological consequences of recent climate change. Conservation Biology (15), 320-331.

Michelozzi, P., de Donato, F., Bisanti, L., Russo, A., Cadum, E., DeMaria, M., D'Ovidio, M., Costa, G. \& Perucci, C. (2005). The impact of the summer 2003 heat waves on mortality in four Italian cities. Euro Surveill, 10(7), 61-165.

Myung, H. N. \& Jang, J. Y. (2011). Causes of death and demographic characteristics of victims of meteorological disasters in Korea from 1990 to 2008. Environmental health, 1o(82), 145-173. DOI: http://doi.org/10.1186/1476-069X-10-82

Noji, E. (200o) Naturaleza de los desastres: sus características generales y sus efectos en la salud pública. En OPS (Ed.), Impacto de los Desastres en la Salud Pública. Organización Panamericana de la Salud. Bogotá: OPS. Recuperado de: http://www.sld.cu/galerias/pdf/sitios/desastres/impacto_de_ los_desastres_en_la_salud_publica_1.pdf [visitado el 12 de oct. 2019]

OPS/OMS. (25 de or de 2019). Organización Panamericana de la Salud/Organización Mundial de la salud. Recuperado de: https://www.paho.org/hq/index.php?option=com_content\&view=article\& id=3564:2010-health-information-analysis\&Itemid=3644\&lang=es [visitado el 12 de oct. 2019]

Reckien, D. (2018). What is in an index? Construction method, data metric, and weighting scheme determine the outcome of composite social vulnerability indices in New York City. Regional Environmental Change, 18(5), 1-13.

Riojas Rodríguez, H. \& Hurtado-Díaz, M. (2006). Estudio diagnóstico sobre los efectos del cambio climático en la salud humana de la población en México. Ciudad de México: Instituto Nacional de Salud Pública.

Schmidtlein, M.C., Deutsch, R.C., Piegorsch, W.W. \& Cutter, S. L. (2008). A Sensitivity Analysis of the Social Vulnerability Index. Risk Analysis, 28(4), 1099-1114

SSA. (18 de 03 de 2019). Secretaría de Salud. Recuperado de: http://www.dgis.salud.gob.mx/contenidos/ difusion/cdefuncion.html [visitado el 12 de oct. 2019]

Tate, E. (2012). Social vulnerability indices: a comparative assessment using uncertainty and sensitivity analysis. Nature Hazards (63), 325-347.

Vincent, K. (2004). Creating an index of social vulnerability to climate change for Africa. University of East Anglia. Anglia: Tyndall Center for Climate Change Research.

Zagheni, E., Muttarak, R. \& Striessnig, E. (2016). Differential mortality patterns from hydrometeorological disasters: Evidence from cause-of-death data by age and sex. Vienna Yearbook of Population Research (13), 47-70. 